\title{
Understanding aggregation induced emission in a propeller-shaped blue emitter
}

\author{
Dr Ljiljana Stojanović and Dr Rachel Crespo-Otero*
}

\section{Abstract}

Organic fluorophores with an enhanced emission in the condensed phase have great potential for the design of optoelectronic materials. Several propellershaped molecules show aggregation-induced emission (AIE), in particular, silole derivatives have attracted significant attention because of their significant quantum yields in the solid state. In this contribution, we investigate the mechanism of AIE of a propeller-shaped blue emitter: 1,2,3,4-tetraphenyl-1,3-cyclopentadiene (TPC). We explore the excited state mechanism in the light of models most commonly used to explain it: restriction of intramolecular motions (RIM) and restricted access to the conical intersection (RACI). Our interpretation is supported by excited state dynamics simulations and the analysis of Huang-Rhys factors and reorganisation energies. We quantify the effects of intermolecular interactions and exciton couplings. The mechanism for TPC is compared with previous investigations of analogue silole compounds. Our systematic investigation highlights the role of conical intersections on the nonradiative decay mechanisms and complementary descriptions provided by the RIM and RACI models.

\section{Introduction}

Highly emissive organic materials find applications in organic light-emitting diodes (OLEDs), photovoltaic cells, solid-state lasers and biological sensors. Designing deep blue organic emitters has been particularly challenging due to their low emission efficiency and poor stability.[1] Another major challenge for the design of efficient materials is the aggregation-caused quenching induced by stabilisation of $\pi \pi^{*}$ and charge transfer aggregates promoting deactivation through nonradiative pathways. Several molecules exhibiting an enhanced emissive response in the condensed phase, aggregation-induced emission (AIE), have been reported in the last years.[2]

* School of Biological and Chemical Sciences, Queen Mary University of London, Mile End Road, London E1 4NS, UK, e-mail: r.crespo-otero@qmul.ac.uk 
The first described AIE fluorophores were silole molecules with a propellershaped structure (Figure 1).[3] These compounds can show very high quantum yields in the solid state, for example, the quantum yields of MPPS and DMTPS are 0.76 and 0.85 in solid films.[4] These molecules have an extended electron delocalisation involving the $\pi$ cloud from the butadiene moiety and the $\sigma^{*}(\mathrm{Si}-\mathrm{C})$ orbital. Consequently, they usually emit in the green and yellow, but rarely in the blue.[5] A strategy to obtain blue emitters is to replace the $\mathrm{Si}$-atom by a $\mathrm{C}$-atom. Because of the strong $s p^{3}$-hybridisation of the C-atom, the $\sigma-\pi$ conjugation is reduced, which blueshifts emission with respect to the silole derivatives.

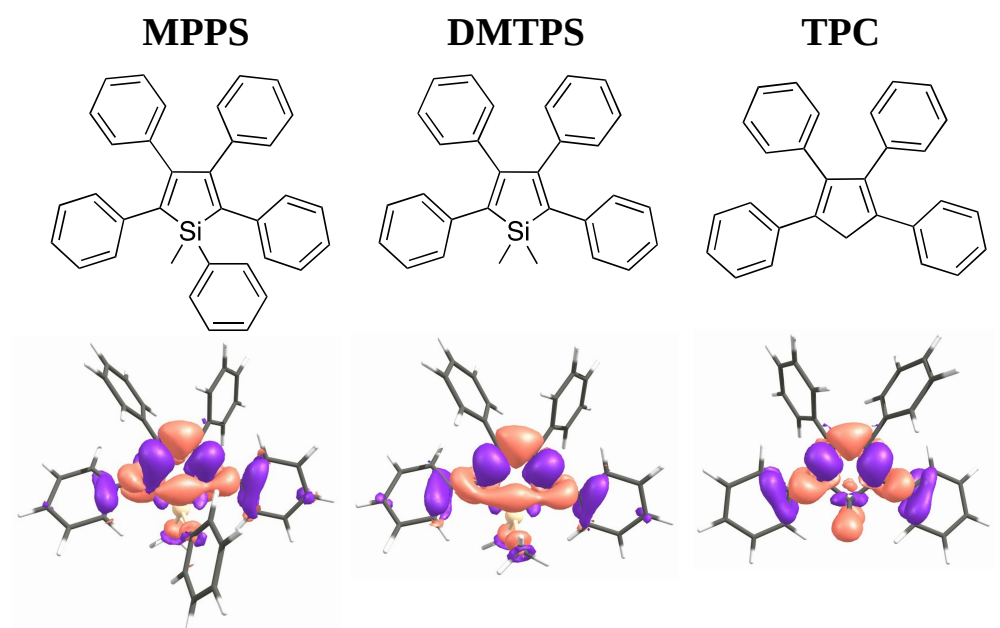

Figure 1: Chemical structure of some AIE propeller-shaped molecules. MPPS:1-methyl-1,2,3,4,5-pentaphenylsilole, DMTPS: 1,1-dimethyl-1,2,3,4tetraphenylsilole, TPC:1,2,3,4-tetraphenyl-1,3-cyclopentadiene. The $\mathrm{S}_{1}-\mathrm{S}_{0}$ densities show the more extended delocalisation of the electron densities for the silole compounds MPPS and DMTPS.

1,2,3,4-tetraphenyl-1,3-cyclopentadiene (TPC, Figure 1) has a low fluorescence quantum yield in organic solvents that increases in solid films.[6] The quantum yield in solid films with respect 1,2,3,4,5-pentaphenyl-1,3-cyclopentadiene, another AIE chromophore, is 0.37. [7] Derivatives of TPC are efficient blue emitters showing mechanochromic properties and AIE.[5] Because of their properties, these molecules have been suggested as candidates for luminous layer material in single- and multi-layered organic electroluminescence devices in several patents (for example in reference [8]). While the excited state mechanisms of silole derivatives have been explored by different groups, [9] the mechanisms behind AIE in TPC compounds have not been extensively explored. Herein, we analyse the AIE mechanism of TPC considering the complementary de- 
scriptions provided by the two main models traditionally used to explain AIE: restriction of inter-molecular motions (RIM) and restricted access to the conical intersection (RACI).[10] According to the RIM model, the AIE mechanism is associated with a restriction of low-frequency vibrations, which are essential in nonradiative decay. Based on Fermi's golden rule, Shuai et al. have provided a theoretical framework for the interpretation of this model.[11] An alternative is the RACI model proposed by Li and Blancafort [9, 12, 10], which considers the significant role of conical intersections as funnels of electronic excitations. In the condensed phase, the energy of the $\mathrm{S}_{1}-\mathrm{S}_{0}$ conical intersections can increase because of steric hindrance blocking nonradiative deactivation pathways and enhancing the emissive response.

In this paper, we present an analysis of the main radiative and nonradiative mechanisms of TPC based on the investigation of the excited states energy surfaces in the vacuum, solution, and crystal phase. We investigate the effect of intermolecular interactions and exciton formation in the molecular crystal. Excited state dynamics simulations and the analysis of the Huang-Rhys factors showed the role of vibrations in the excited state mechanisms. We also identify the conical intersections associated with the nonradiative decay processes. We believe our results provide a detailed understanding of the photochemistry of TPC complementing previous investigations on propeller-shaped fluorophores.

\section{Computational Details}

To investigate the AIE mechanism, we considered the excited states of TPC in the gas phase, in acetonitrile solvent, and solid state. The ground state $(\mathrm{FC})$ and $\mathrm{S}_{1}$ minima were optimised at $\omega \mathrm{B} 97 \mathrm{X}-\mathrm{D} / 6-31 \mathrm{G}(\mathrm{d})$ level [13] within the (TD-)DFT frameworks. $[14,15,16,17,18]$ We assessed the performance of several electronic structure methods for the prediction of the absorption and emission energies. B3LYP/6-31G(d) level of theory was also used to model TPC excited states. The polarisable continuum model (PCM) allowed for the consideration of the dielectric environment of the acetonitrile solvent $(\epsilon=35.688)$.

We also used resolution-of-the-identity coupled-cluster with approximate second-order excitations (RI-CC2),[19, 20, 21, 22] and complete active space perturbation theory (CASPT2) method.[23, 24, 25] The CASPT2 computations were based on configuration state functions obtained with $\operatorname{SA}-2-\operatorname{CASSCF}(10,10) .[26]$ The active space included $\pi$ orbitals with significant occupations (Supporting Information). These calculations were done without an IPEA shift and applying an imaginary shift of $0.1 \mathrm{au}$, which solves the problem of intruder states with a negligible effect on the properties of the system.[27] Since the wave-functionbased methods were found to be more sensitive to the basis set choice, we used aug-cc-pVDZ for CC2, CASSCF and CASPT2 calculations. For CASPT2 computations, the second set of d-orbitals of carbon was removed from aug-cc-pVDZ set (we denote this set as (aug-)cc-pVDZ). The $\mathrm{S}_{1}-\mathrm{S}_{0}$ minimum energy conical intersections (MECIs) were optimised with the SA-2-CASSCF $(10,10) / 6-31 \mathrm{G}(\mathrm{d})$ level of theory, using the branching plane update method[28] implemented in 
Molcas (OpenMolcas v18.0 binaries).[29] The RI-CC2 computations were performed with the Turbomole v7.0 code.[30]

The experimental crystal structure of TPC, retrieved from the Cambridge Crystallographic Database (the CCDC code is 1275269),[31] was refined with periodic boundary conditions DFT calculations as implemented in the Quantum Espresso code.[32] The PBE-D2 functional was used with a plane-wave cutoff of 30 Ry and a Monkhorst-Pack k-point grid of $(1 \times 2 \times 1)$ in accordance with the shape of the unit cell.

A cluster composed of 45 TPC molecules (2295 atoms) was extracted from the optimised supercell. This cluster model was used in QM:MM calculations with electrostatic embedding. A single molecule was treated within the QM framework, whereas the surrounding molecules represented by the molecular cluster were treated using a MM method. The QM region was relaxed whilst the MM region was kept fixed at its optimised lattice positions.

FC and $\mathrm{S}_{1}$ geometries were optimised applying the $\mathrm{ONIOM}(\mathrm{QM}: \mathrm{MM})$ method $[33,34]$ using the Gaussian 16 software. [35] The QM region was treated using the $\omega \mathrm{B} 97 \mathrm{X}-\mathrm{D} / 6-31 \mathrm{G}(\mathrm{d})$ level of theory under the (TD-)DFT framework. The MM region was simulated with the Amber force field[36] using ESP charges derived from a vacuum $\mathrm{HF} / 3-21 \mathrm{G}^{*}$ calculation of the monomer. The $\mathrm{S}_{1}-\mathrm{S}_{0}$ MECI in the solid state was optimised using the QM/MM method. The QM region was described at the SA-2-CASSCF $(10,10) / 6-31 G(d)$ level of theory, whereas the surrounding cluster was treated using the Amber force field. The $\mathrm{S}_{1}-\mathrm{S}_{0}$ MECI was optimised using the interface between the Molcas and Tinker (version 6.3.3) codes.[29]

Exciton couplings $(J)$ were computed applying a diabatisation scheme based on the transition dipole moments of the isolated molecules and the dimers[37, 38]. This method takes into account short-range (exchange, orbital overlap, charge-transfer) and long-range (Coulomb) interactions. The Huang-Rhys factors and reorganisation energies were computed for the molecules in gas phase, solution, and solid state based on the $\mathrm{S}_{1}$ and $\mathrm{S}_{0}$ minima and the normal modes computed at (TD-) $\omega$ B97X-D/6-31G(d) level using the Dushin code.[39] Exploratory adiabatic dynamics was performed to follow the initial relaxation of TPC in the $\mathrm{S}_{1}$ state using the Newton-X code.[40, 41, 42] These simulations were performed using the TD- $\omega$ B97X-D/6-31G(d) level of theory. Initial conditions were generated from the simulated absorption spectra.[43] 20 trajectories were simulated starting from the $\mathrm{S}_{1}$ state. They were propagated for a maximum of $500 \mathrm{fs}$ with a time step of $0.5 \mathrm{fs}$. A Wigner distribution of 500 geometries was generated based on the vibrational frequencies of $\mathrm{S}_{1}$ and used to simulate the emission spectra of $\mathbf{T P C}$ in the solution and crystal applying the nuclear ensemble approach as implemented in Newton-X.[43, 44]

\section{Results and Discussion}

The enhancement of fluorescence witnessed in the TPC crystal with respect to solution can be originated from an increment of the radiative decay $\left(k_{r}\right)$ or/and 
a decrease of nonradiative decay rates $\left(k_{n r}\right)$ as a result of aggregation.[12, 45] In the next sections, we analyse how these mechanisms are affected in the solid state considering different factors and models.

\subsection{Vertical excitation and radiative mechanisms}

Table 1 shows the vertical excitations for the Franck Condon (FC) geometry and the emission energies of TPC at different levels of theory. We considered single reference (TD-DFT and CC2) and multireference methods (CASPT2/CASSCF). The CASPT2 and CC2 excited states were computed using the geometries optimised with (TD-) $\omega$ B $97 \mathrm{X}-\mathrm{D} / 6-31 \mathrm{G}(\mathrm{d})$.

Table 1: Vertical and adiabatic excitation energies and oscillator strengths (in parenthesis) of the $\mathrm{S}_{1}$ state of TPC molecule in vacuum, solution (acetonitrile), and crystal environment.

\begin{tabular}{ccccc}
\hline & \multicolumn{5}{c}{ Energy $(\mathrm{eV})$} \\
\hline & \multicolumn{3}{c}{ Vacuum/Solution } & \multicolumn{2}{c}{ Crystal } \\
& Absorption & Emission & Absorption & Emission \\
\hline RI-CC2/aug-cc-pVDZ & $3.76(0.42)$ & $2.85(0.51)$ & $3.72(0.58)$ & $2.87(0.50)$ \\
TD-B3LYP/6-31G(d) & $3.37(0.34)$ & $2.54(0.35)$ & $3.39(0.50)$ & $2.55(0.45)$ \\
TD- $\omega$ B97X-D/6-31G(d) & $3.89(0.36)$ & $2.78(0.41)$ & $3.85(0.52)$ & $2.75(0.46)$ \\
TD- $\omega$ B97X-D/6-31G(d), PCM & $3.85(0.45)$ & $2.57(0.62)$ & - & - \\
MS-2-CASPT2/(aug-)cc-pVDZ & 3.98 & 3.08 & 3.91 & 3.05 \\
Experimental[7] & 3.62 & 2.85 & 3.44 & 2.75 \\
\hline
\end{tabular}

The absorption spectrum of $\mathbf{T P C}$ in solution and in crystal is dominated by the $\mathrm{S}_{1}-\mathrm{S}_{0}\left(\pi \pi^{*}\right)$ transition. The vertical excitations to $\mathrm{S}_{1}$ obtained with RI-CC2 and TD- $\omega$ B97X-D in the vacuum are overestimated with respect to the experimental values in solution $(0.14 \mathrm{eV}$ and $0.27 \mathrm{eV}$ respectively), while the value with TD-B3LYP is underestimated by $0.28 \mathrm{eV}$. Emission energies obtained with these methods show similar shifts. The largest deviations from the experimental values are obtained with MS-2-CASPT2/(aug-)cc-pVDZ, however this is still reasonable considering the fact that we are using a $(10,10)$ active space.

For both absorption and emission, the environment has a small effect on the energy gaps. Going from the gas phase to solution, the absorption energy shifts by only $0.04 \mathrm{eV}$ at the TD- $\omega \mathrm{B} 97 \mathrm{X}-\mathrm{D} / 6-31 \mathrm{G}(\mathrm{d})$ level of theory. The crystal environment shifts the absorption energy for an amount of $0.04 \mathrm{eV}$ and while emission is shifted $0.03 \mathrm{eV}$. Emission is more affected in acetonitrile (dielectric constant $=35.7$ ), with a shift of $0.21 \mathrm{eV}$. In general, all tested methods reproduced vertical and emission energies reasonably well. The agreement between calculated and experimental data for the solid is very good. However, we should take into account that the experimental values are reported for solid films that could feature amorphous or polycrystalline morphologies[7] and our 
model is based on a perfect crystal. Given the small polarity of the crystal, composed only of Carbon and Hydrogen, a weakly dependence with the crystal environment seems to be reasonable.

We simulated the emission spectra in solution and crystal using the TD$\omega \mathrm{B} 97 \mathrm{X}-\mathrm{D} / 6-31 \mathrm{G}(\mathrm{d})$ level of theory (Figure 2). The spectra are characterised by maxima at $2.58 \mathrm{eV}$ and $2.74 \mathrm{eV}$ in solution and the crystal phase respectively. This represents a very small shift with respect to the vertical emission energies (Table 1).

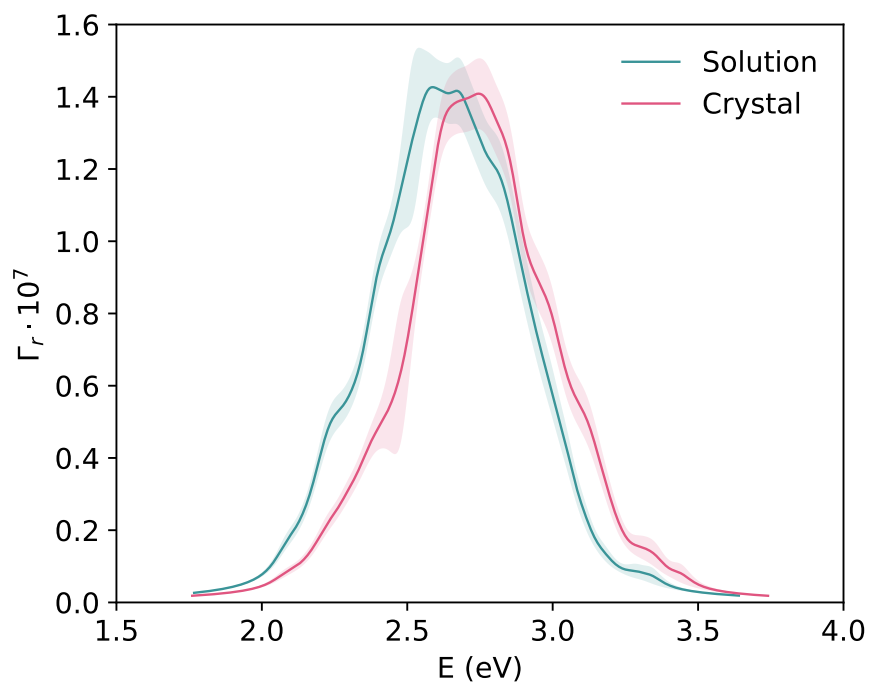

Figure 2: Simulated emission spectra of TPC in solution and crystal. The excitation energies were convoluted with Lorentzian line shapes with a broadening of $0.10 \mathrm{eV}$. The shaded areas represent errors in the numerical integration.

Based on the simulated emission spectrum (Figure 2, given as the differential rate of spontaneous photon emission $\left(\Gamma_{r}\right)$ ) with respect to the emission energies, we calculated the radiative rates by numerical integration considering the following equation:[44]

$$
k_{r}=\frac{1}{\hbar} \int \Gamma_{r}(E) d E
$$

The $k_{r}$ values were $1.44 \times 10^{8} \mathrm{~s}^{-1}$ in solution and $1.35 \times 10^{8} \mathrm{~s}^{-1}$ in crystal. They are in agreement with the reported ones for related propeller-shaped molecules. [46, 47] The radiative rates obtained for both phases are very similar, the value for solution is slightly larger than the solid state rate. This highlights that the mechanisms for the enhancement of fluorescence should be related with a restriction of nonradiative mechanisms (see discussion in the next sections). 


\subsubsection{Crystal packing: intermolecular interactions and exciton cou- plings}

Intermolecular interactions play a significant role on excited state mechanisms, they affect radiative and nonradiative decay pathways.[10] Exciton coupling controls the delocalisation of the electronic density and regulates electron transport in molecular crystals. Herein, we quantify the role of intermolecular interactions and exciton couplings in the TPC crystal.

The propeller shape of the TPC molecule does not allow for an effective packing and the crystal has a relatively low density. We extracted all dimers (5) with distances between the centroids of TPC smaller than $10 \AA$ and calculated their exciton couplings (D1-D5, Figure 3). The main intermolecular interactions found in the TPC crystal are weak C-H.. $\pi$, C-H..C, and H..H interactions. The transition density differences in all dimers are located on the cyclopentadiene moieties of the constituent monomers (Supporting Information), mirroring the behaviour of the single monomer depicted in Figure 1. Because of the small overlap of the electronic densities, all dimers experience weak exciton couplings.

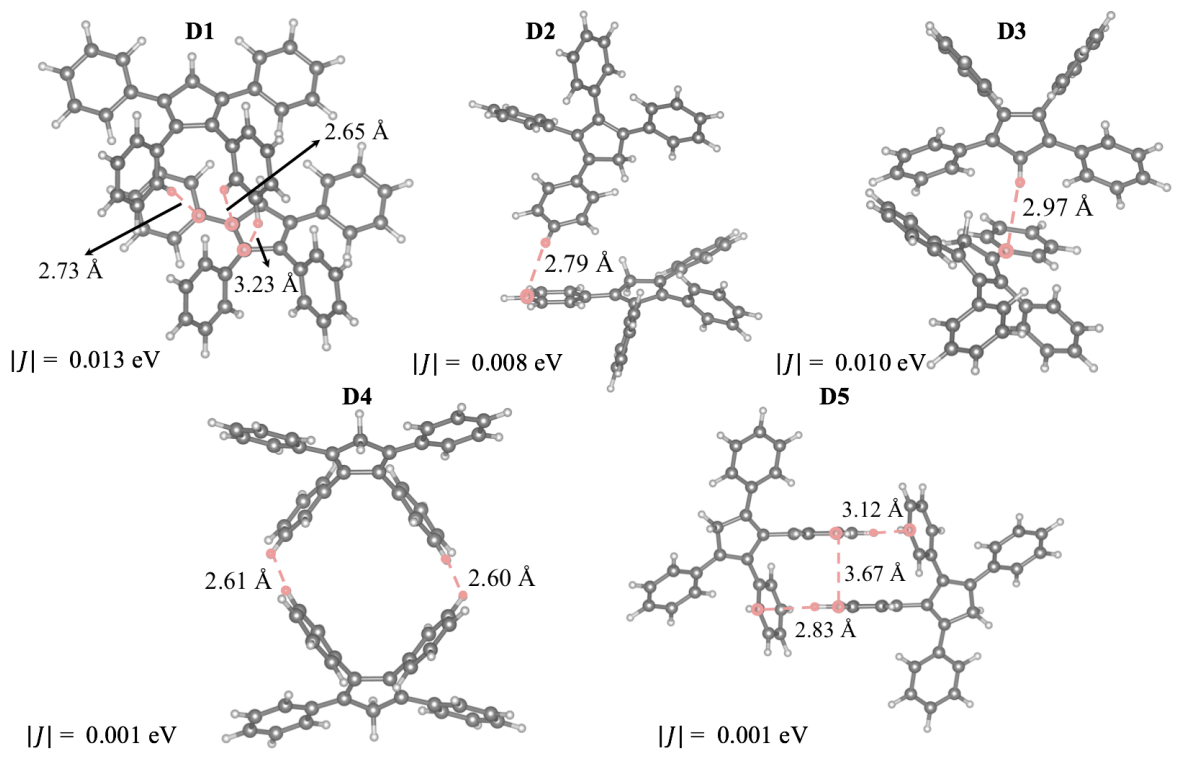

Figure 3: Structures of dimers in the TPC showing closest intermolecular contacts $(\AA)$ and absolute values of the exciton couplings (in $\mathrm{eV}$ )

The five dimers can be approximately classified according to the Kasha's excitonic model as H-dimers [48]. The bright state of all the dimers is $\mathrm{S}_{2}$. The oscillator strengths of $\mathrm{S}_{1}$ for the dimers D1, D4, and D4 are negligible and their values of $\mathrm{S}_{2}$ are almost twice those of the isolated molecules (Supporting Information). For D2 and D3, the oscillator strengths of $\mathrm{S}_{1}$ are 0.22 and 0.27 respectively. The dimer with the largest coupling, of $0.013 \mathrm{eV}$, is $\mathbf{D} \mathbf{1}$, which is 
characterised by the short interatomic contacts, which are C-H..C distances of $2.65,2.73$, and $3.23 \AA$. D4 and D5 dimers have very small $J$ values (0.001), as a consequence of the large spatial separation of cyclopentadiene rings. Dimer D5 features a $\pi . . \pi$ interaction at $3.67 \AA$, which does not significantly contribute to the exciton coupling. Dimer D4 has a closed structure with the two molecules interacting via two H..H contacts at distances of $2.6 \AA$.

Exciton couplings are of similar order to those obtained for 1,1,2,3,4,5hexaphenylsilole $(0.009-0.013 \mathrm{eV})$.[49] Their small values in comparison to the reorganisation energies (see next section) indicates that electron transport will mainly be incoherent and localised, similarly to well-known organic crystals like anthracene.[37] In ideal Kasha H-dimers, the emission is quenched because radiative decay is forbidden.[48] However, this model cannot properly represent dimers where the interaction between the molecular densities are not well described by the point-dipole point-dipole approximation.[10] Additionally, aggregation can significantly alter nonradiative mechanisms. In a recent paper by Shuai et. al [49], the authors found that regardless of the nature of the investigated dimers ( $\mathrm{H}$ or J aggregates), exciton couplings always enhance nonradiative decay constants, however, the effect is very small for low couplings such as those reported here. Given the loose nature of the Frenkel excitons in TPC, exciton couplings should not have a significant role in the excited state mechanisms.

Recent research has highlighted the possible role of through-space $\pi . . \pi$ interactions in the AIE mechanism of molecular rotors. [50] The analysis of intermolecular interactions in the TPC crystal does not show significant intermolecular $\pi . . \pi$ interactions. We have found that in the three phases considered in our calculations, electronic excitation involves mainly the central aromatic moiety and no electron transfer between vicinal aromatic rings was observed. Consequently, intramolecular $\pi . . \pi$ interactions do not seem to be essential for the AIE mechanism.

\subsection{Relaxation in the excited state}

\subsubsection{Reorganisation energies}

According to the RIM model, for AIE fluorophores, low-frequency modes are hindered in the solid state. $[3,6]$ To analyse the effect of aggregation on the vibrations, we calculated the Huang-Rhys factors $\left(S_{j}\right)$ and reorganisation energies $(\lambda)$ of TPC in vacuum, solution, and the solid state. The $S_{j}$ factors are shown in Figure 4 . The $\lambda$ values are obtained by summing the $\hbar \omega_{j} S_{j}$ contributions of the normal modes with frequencies $\omega_{j}$, according to:

$$
\lambda=\sum_{j} \hbar \omega_{j} S_{j}
$$

We decomposed the total reorganisation energy into the contributions of three different kinds of normal modes - torsional, bending, and stretching (Table $2)$. For the $S_{1}-S_{0}$ transition, the total $(\lambda)$ values are $5288 \mathrm{~cm}^{-1}(0.66 \mathrm{eV}), 5978$ 
$\mathrm{cm}^{-1}(0.78 \mathrm{eV})$, and $4263 \mathrm{~cm}^{-1}(0.53 \mathrm{eV})$ for vacuum, solution, and solid state, respectively (Table 2 ).
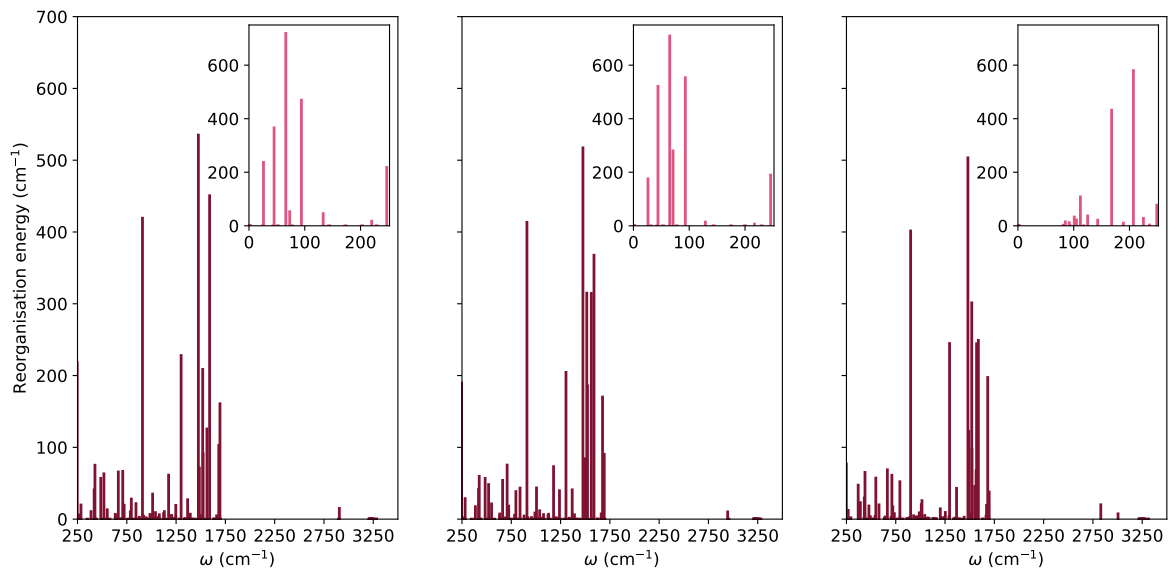

Figure 4: Reorganisation energies $\left(\mathrm{cm}^{-1}\right)$ for the $\mathrm{S}_{1}-\mathrm{S}_{0}$ transition in, from left to right, gas phase, solution, and solid state.

Table 2: Reorganisation energies (in $\mathrm{cm}^{-1}$ ) for $\mathrm{S}_{1}-\mathrm{S}_{0}$ transition decomposed into the contributions of torsional, bending, and stretching modes.

\begin{tabular}{cccc}
\hline & \multicolumn{3}{c}{ Energy $\left(\mathrm{cm}^{-1}\right)$} \\
\hline & Gas & Solution & Crystal \\
\hline Torsional & 2185 & 2493 & 1425 \\
Bending & 2135 & 2508 & 2294 \\
Stretching & 967 & 976 & 903 \\
Total & 5288 & 5978 & 4623 \\
\hline
\end{tabular}

In vacuum and solution, the larger contributions to the total reorganisation energies are the torsional and stretching modes, whereas bending modes contribute $\approx 20 \%$ to the total reorganisation energy. In the crystal, the main components are the bending vibrations $(\approx 50 \%)$. There are three modes with large contributions to the reorganisation energies which show a similar behaviour in gas phase, solution and the molecular crystal (914.64, 1476.91 and $1588.60 \mathrm{~cm}^{-1}$, Figure 5). These collective vibrations will be activated as a result of the bond alternation and conformational change of the aromatic rings required in the electronic transitions.

In line with previous investigations of the AIE mechanisms in MPPS and DMTPS, $[47,4]$ aggregation has a significant effect on the low-frequency region (Figure 4). In contrast with vacuum and solution, frequencies smaller than 150 
$\mathrm{cm}^{-1}$ have a very small contribution to the reorganisation energies in the solid state. Consequently, there is a decrease in $\lambda$, which is mainly associated with the reduction of torsional contributions. In particular the out-of-plane rotation of the phenyl rings with respect to cyclopentadienyl ring are hampered because of geometrical restrictions imposed by the packing (Figure 8). The restriction of these motions is essential for the enhancement of the emission in the solid state.

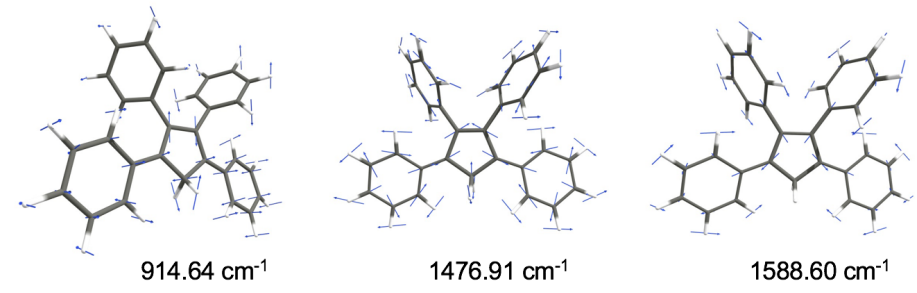

Figure 5: Modes with significant contributions to the reorganisation energies for $\mathrm{S}_{1}-\mathrm{S}_{0}$ transitions in the three phases.

\subsubsection{Excited state dynamics}

Trajectory based dynamics simulations can provide information about excited state relaxation and have been previously employed to explore AIE fluorophores. [51, 52] Herein, we explore the initial relaxation of TPC in the $S_{1}$ state. We propagated 500 fs of dynamics of the TPC using TD- $\omega$ B97X-D/6-31G(d) level of theory. The aim of these simulations was to complement the description provided by the vibrational analysis.

We used normal mode analysis $[53,54]$ to decompose nuclear motions into the normal modes taking the FC geometry as a reference. In this method, the trajectories are averaged to remove random displacements. [53] To detect which vibrations were significantly activated during the dynamics, we analysed the standard deviation of normal mode displacements in five intervals of time (0-100, 101-200, 201-300, 301-400, and 401-500 fs). The modes with the most significant coherent behaviour are shown in Figure 6.

The vibrational mode showing the most significant activation in the first 100 fs corresponds to a frequency of $233 \mathrm{~cm}^{-1}$. This mode involves an in-plane distortion of the cyclopentadiene ring (Figure 8). In the higher energy region, collective C-C stretching vibrations (modes around $1523 \mathrm{~cm}^{-1}$ ) are also activated. The transition from $\mathrm{S}_{0}$ to $\mathrm{S}_{1}$ triggers a redistribution of the electronic density of TPC, which involves bond alternation in the central cyclopentadiene ring. The bond patterns in $S_{1}$ and $S_{0}$ can be understood taking by considering the contributions of different resonant structures to the electronic density (Figure 7). The alternation of bonds can be visualised from the analysis of the $\mathrm{S}_{1}-\mathrm{S}_{0}$ electron densities (Figure 1 ). 


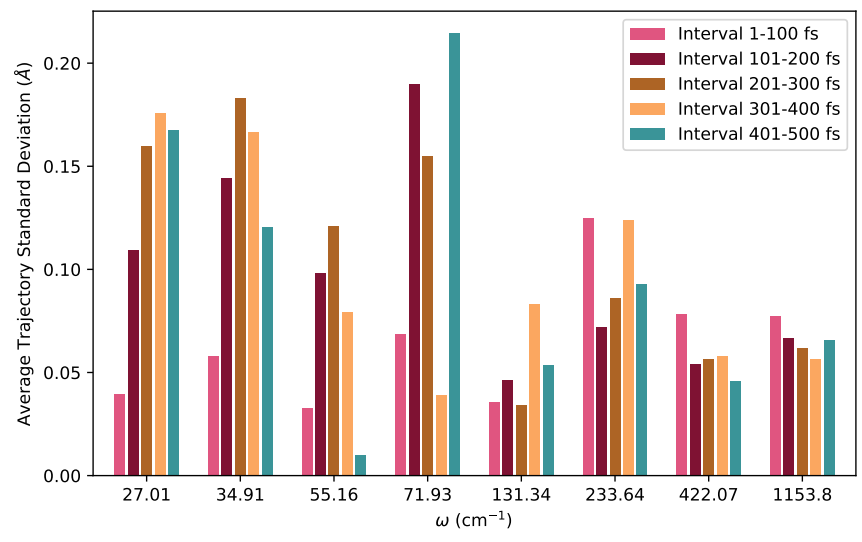

Figure 6: Total standard deviation of each normal mode for an averaged trajectory in different time intervals. Modes are only shown if their mean standard deviation during $500 \mathrm{fs}$ is larger than $0.07 \AA$.

In $\mathrm{S}_{0}$, the larger contribution to the electronic density comes from the typical cyclopentadiene resonant structure $\mathbf{I}$. However, the $\mathrm{S}_{1}$ state is better described by a biradical structure, where $\mathbf{R} \mathbf{1}$ and $\mathbf{R 3}$ become single bonds and $\mathbf{R 2}$ a double bond (II, Figure 7). This structure is stabilised by delocalisation of the radicals over the phenyl rings, which is maximised by the planarisation of the two adjacent phenyl rings in $\mathrm{S}_{1}$. The dihedral angles decrease from $36^{\circ}$ in the $\mathrm{FC}$ region to $9^{\circ}$ in the $\mathrm{S}_{1}$ minimum. Thus, the low-frequency torsional modes of phenyl rings $\left(\omega_{j}<100 \mathrm{~cm}^{-1}\right)$ show the most significant coherence with increasing activation during dynamics (Figure 8). These modes correspond to those restricted in the solid state according to the analysis of the reorganisation energies (Figure 4 and Supporting Information).

The alternation bond coordinate defined as $d=\mathbf{R} \mathbf{1}+\mathbf{R} 3-\mathbf{R} 2$ can be followed in the dynamics simulations. Figure 7 shows the value of $d$ averaged over all trajectories. $d$ increases promptly during the first $15 \mathrm{fs}$ starting from the value in the ground state $(\mathrm{d}=1.27 \AA)$ to $1.65 \AA$. Then, bond alternation coordinate decreases and begins an oscillatory motion around the $\mathrm{S}_{1}$ minimum $(\mathrm{d}=1.46 \AA)$ after 30 fs of dynamics (Figure 7).

We will show in the next section that the main nonradiative mechanism of TPC is ring distortion through puckering, which is in line with the mechanisms for DMTPS.[12] The modes associated with the out-of-plane bending of the cyclopentadiene ring leading to the corresponding conical intersection are not significantly activated during the first 500 fs of dynamics. However, nonradiative decay through puckering conical intersections normally takes longer than 500 fs.[55] We should also take into account that the mechanism cannot 
I
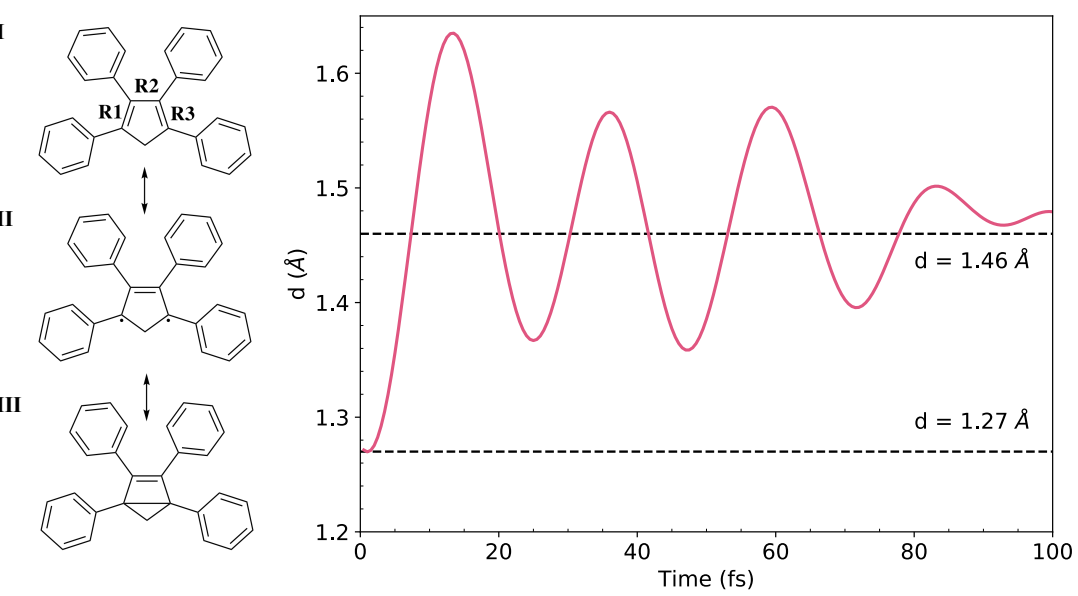

Figure 7: Time evolution of the bond alternation coordinate $(d=\mathbf{R} 1+\mathbf{R} 3-\mathbf{R 2})$ during first 100 fs of dynamics.

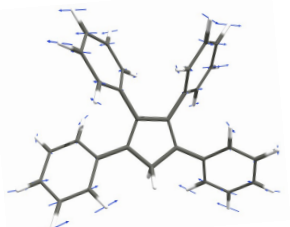

$27.01 \mathrm{~cm}^{-1}$

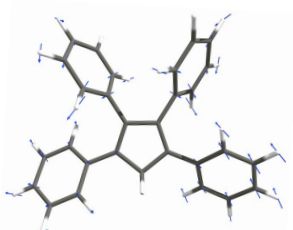

$71.93 \mathrm{~cm}^{-1}$

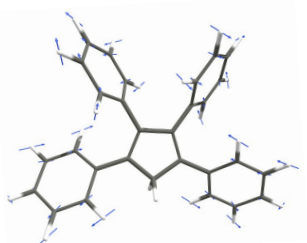

$34.91 \mathrm{~cm}^{-1}$

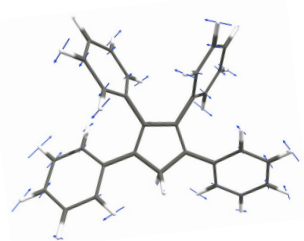

$131.34 \mathrm{~cm}^{-1}$

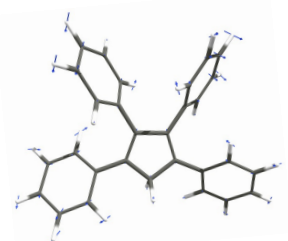

$55.16 \mathrm{~cm}^{-1}$

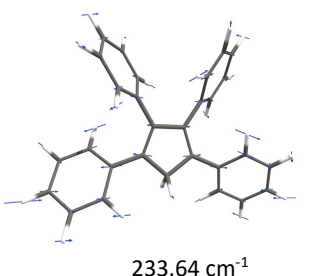

$233.64 \mathrm{~cm}^{-1}$

Figure 8: Low energy vibrations with significant activation in the dynamics. 
be naturally described by TD-DFT methods. [56] For example, according to the TD- $\omega$ B97X-D results, the $\mathrm{S}_{1}-\mathrm{S}_{0}$ puckered MECI is located at $\approx 0.6 \mathrm{eV}$ above the provided excitation energy. To explore the later steps of the mechanism, we used multireference calculations.

\subsection{Relaxation mechanisms}

The previous sections have focused on the investigation of the initial relaxation of TPC in vacuum. In this section, we explore the excited state mechanisms under the RACI model. We optimised the $\mathrm{S}_{1}-\mathrm{S}_{0}$ MECI geometries using SA-2CASSCF $/ 6-31 G(d)$ in the vacuum and solid state. The MECI geometries correspond to cyclopentadiene ring puckering with additional flapping motions of phenyl substituents (Figure 9) and are similar to the arrangements reported for DMTPS [12], cyclopentadiene, and 1,2,3,4-tetramethyl-cyclopentadiene[54]. In the vacuum, four atoms of the central molecule remain almost in plane $(\mathrm{C} 1-\mathrm{C} 2-$ C3-C4, $\alpha=2.9^{\circ}$ ), while the fifth carbon (C5) is puckered (C3-C4-C5-C6, $\beta=$ $\left.108.1^{\circ}\right)$.

In the solid state, the molecule distorts, decreasing the repulsion with the environment. The $\alpha$ dihedral deviates $11.2^{\circ}$ from the plane to allow for the increase of the $\beta$ angle $\left(138.7^{\circ}\right)$. As a result, $\mathrm{S}_{1}-\mathrm{S}_{0}$ MECI in the solid state is more compact than the structure in vacuum and also more energetic. The puckering of C5 significantly reduces the C5-C2 distance, which becomes 2.09 and $1.94 \AA$ in vacuum and the solid state respectively. In DMTPS, the values of these distances are 2.57 and $2.26 \AA$ in solution and solid state.[12]
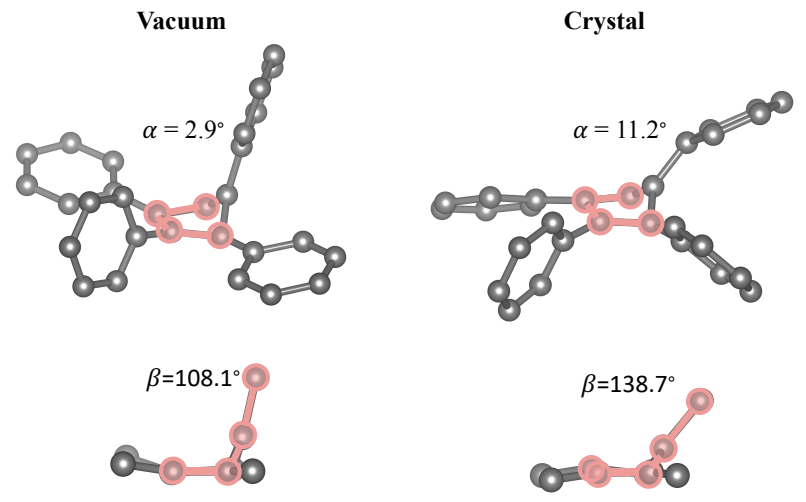

Figure 9: Geometries of the optimised $\mathrm{S}_{1}-\mathrm{S}_{0} \mathrm{MECI}$ in vacuum (left) and crystal (right) viewed from two different angles.

In the $\mathrm{S}_{1}-\mathrm{S}_{0} \mathrm{MECI}$, there is a significant contribution of resonance structure III (Figure 7) and the central cycle in the $\mathrm{S}_{1}-\mathrm{S}_{0}$ MECI resembles the structure of bicyclo $[2,1,0]$ pentene. Consequently, there is an increase of the electronic 


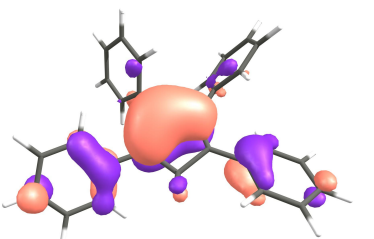

FC

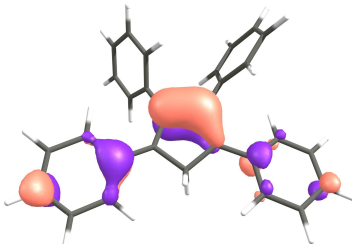

$\mathrm{S}_{1}$ minimum

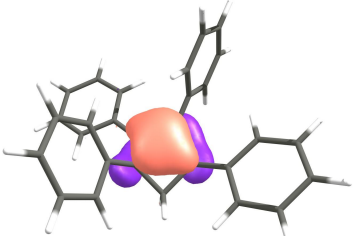

$\mathrm{MECl}$

Figure 10: $\mathrm{SA}(2)$-CASSCF $(10,10)$ orbital showing an increasing electronic density between atoms $\mathrm{C} 2$ and $\mathrm{C} 5$.

density in the $\mathrm{C} 2-\mathrm{C} 5$ region as evidenced by an extension of the electronic density in one of the orbitals included in the active space (Figure 10). The occupation of this orbital changes from 1.68 in the $\mathrm{S}_{1}$ minimum to 1.88 in the MECI.

Single point calculations were performed with RI-CC2 and MS-CASPT2 to take into account the effect of electron correlation. Figure 11 shows the energy of $\mathrm{S}_{1}$ and $\mathrm{S}_{0}$ for the FC, $\mathrm{S}_{1}$ minima and the $\mathrm{S}_{1}-\mathrm{S}_{0}$ MECI. The $\mathrm{S}_{1}-\mathrm{S}_{0}$ gap at conicals is not 0 because the geometries were optimised with CASSCF. The gaps are around $0.2 \mathrm{eV}$ and 0.4 for MS-CASPT2 and RI-CC2 respectively. Optimisation of the MECIs at the same level of theory will be required to reduce the gaps. However, this should not change the qualitative picture obtained based on the CASSCF geometries. The energies obtained with SA-2-CASSCF $(10,10) /($ aug)cc-pVDZ are shown in the SI, they provide a similar qualitative interpretation.

As was described in the previous sections, excited-state relaxation in the vacuum first leads to a $S_{1}$ minimum at $3.08 \mathrm{eV}$ where the cyclopentadiene ring is planar. This step should be followed by relaxation to the $\mathrm{S}_{1}-\mathrm{S}_{0}$ MECI. The energetically accessible MECI in the gas phase indicates that internal conversion via puckering $\mathrm{CI}$ is an important relaxation pathway. In the molecular crystal, because the $\mathrm{S}_{1}-\mathrm{S}_{0}$ MECI is classically inaccessible, the relaxation through this mechanism is disfavoured. The $\mathrm{S}_{1}-\mathrm{S}_{0}$ MECI lies $1.6 \mathrm{eV}$ above the initial $\mathrm{S}_{1}$ state and $2.2 \mathrm{eV}$ above the $\mathrm{S}_{1}$ minimum (Figure 11). These calculations show that access to the puckering $\mathrm{S}_{1}-\mathrm{S}_{0}$ MECI is restricted in the solid state, which is consistent with the RACI model.

In the solid state, since decay through puckering is not possible, the TPC will decay to the ground state via fluorescence. Internal conversion facilitated by the overlap between the vibronic levels could be possible provided the molecule stays in the $\mathrm{S}_{1}$ minimum for a long time. However, as shown before, this mechanism is hindered due to the restriction of intramolecular rotations in the solid state. Similarly to MPPS and DMTPS, the enhancement of emission in the solid state can be explained by the restriction of access to nonradiative decay pathways. 

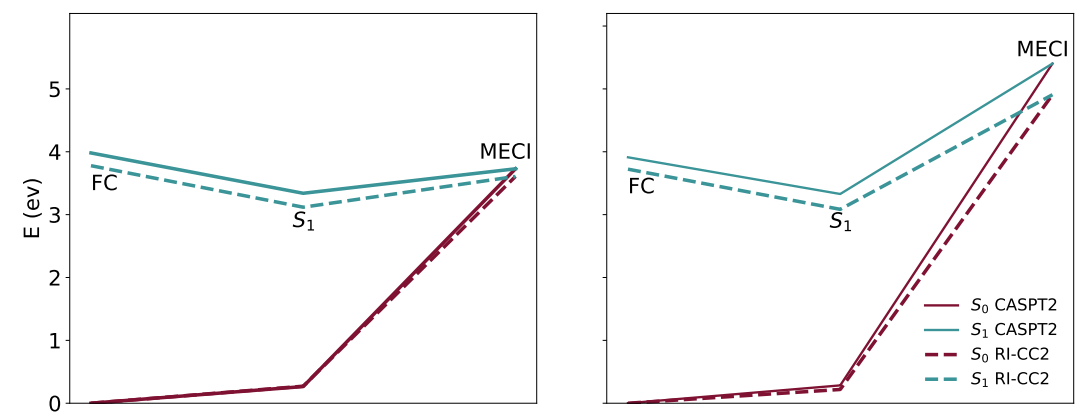

Figure 11: Energies of $S_{0}$ and $S_{1}$ states at FC points, $S_{1}$ minimum, and MECI geometries in vacuum (left) and in crystal (right). The $S_{1}-S_{0}$ MECI energies are represented by averaged $\mathrm{S}_{0}$ and $\mathrm{S}_{1}$ energies.

\section{Conclusions}

In this paper, we investigate the excited state mechanism in $\mathbf{T P C}$, a propellershaped molecule displaying AIE in the solid state. We analysed the potential energy surfaces in the vacuum, solution, and crystal phases as well as the role of vibrations on the excited state mechanisms. Our calculations show that the enhancement of emission in the solid state is associated with the minimisation of decay through nonradiative mechanisms.

The crystal structure shows minimal $\pi-\pi$ interactions and electronic excitations are not significantly perturbed by the crystal environment. However, the TPC molecules are close enough to hinder the vibrations in the excited state with respect to the gas and solution phases. This is clearly shown by both the analysis of Huang-Rhys factors and excited state dynamics. As previously described for similar propeller-shaped systems, the excited state mechanism involves initial relaxation to $\mathrm{S}_{1}$, followed by decay to the ground state through a puckered conical intersection. The access to the conical intersection is restricted in the solid state enhancing the radiative emission.

Our simulations show that the vibrations activated in the initial relaxation are not necessarily those taking the molecule to the minimal energy conical intersections. While the restriction of intramolecular rotations guarantees a less likely decay through traditional internal conversion facilitated by the overlap of vibrational states, if the conical intersections are accessible radiative emission can be quenched. We believe this research helps provide a more complete understanding of AIE in propeller-shaped molecules considering the complementary descriptions provided the RIR and RACI models. 


\section{Acknowledgements}

This research has been supported by the EPSRC (EP/R029385/1). We utilised Queen Mary's Apocrita HPC facility, supported by QMUL Research-IT and the ARCHER UK National Supercomputing Service (EP/L000202) via the Materials Chemistry Consortium and the Thomas cluster via our membership to the Thomas Young Centre. We acknowledge valuable suggestions from Miguel Rivera. We acknowledge the support from the School of Biological and Chemical Sciences at the Queen Mary University of London.

\section{Keywords}

Aggregation-induced emission, blue emitters, computational chemistry, excited states, 1,2,3,4-tetraphenyl-1,3-cyclopentadiene 


\section{Table of Contents}

1,2,3,4-tetraphenyl-1,3-cyclopentadiene shows fast internal conversion in the gas phase due to an accessible conical intersection. In the solid state, this mechanism is hampered, enhancing the fluorescence quantum yield.

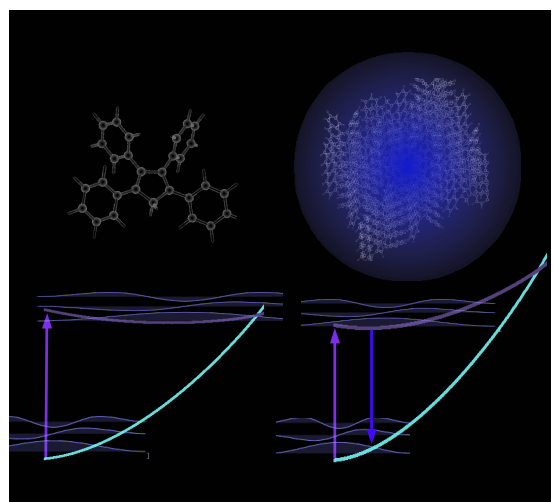




\section{References}

[1] Hungshin Fu, Yi Ming Cheng, Pi Tai Chou, and Yun Chi. Feeling blue? blue phosphors for oleds. Materials Today, 14(10):472-479, 2011.

[2] Ju Mei, Nelson L. C. Leung, Ryan T. K. Kwok, Jacky W. Y. Lam, and Ben Zhong Tang. Aggregation-induced emission: together we shine, united we soar! Chem. Rev., 115(21):11718-11940, 2015.

[3] Yuning Hong, Jacky W. Y. Lam, and Ben Zhong Tang. Aggregationinduced emission. Chemical Society Reviews, 40(11):5361, 2011.

[4] Gui Yu, Shiwei Yin, Yunqi Liu, Jiangshan Chen, Xinjun Xu, Xiaobo Sun, Dongge Ma, Xiaowei Zhan, Qian Peng, Zhigang Shuai, Benzhong Tang, Daoben Zhu, Weihai Fang, and Yi Luo. Structures, electronic states, photoluminescence, and carrier transport properties of 1,1-disubstituted 2,3,4,5-tetraphenylsiloles. Journal of the American Chemical Society, 127(17):6335-6346, 2005.

[5] Zhijun Ruan, Le Li, Can Wang, Yujun Xie, Quanyuan Hu, Qian Peng, Shanghui Ye, Qianqian Li, and Zhen Li. Tetraphenylcyclopentadiene Derivatives: Aggregation-Induced Emission, Adjustable Luminescence from Green to Blue, Efficient Undoped OLED Performance and Good Mechanochromic Properties. Small, 12(47):6623-6632, 2016.

[6] Qi Zeng, Zhen Li, Yongqiang Dong, Chong'an Di, Anjun Qin, Yuning Hong, Li Ji, Zhichao Zhu, Cathy K. W. Jim, Gui Yu, Qianqian Li, Zhongan Li, Yunqi Liu, Jingui Qin, and Ben Zhong Tang. Fluorescence enhancements of benzene-cored luminophors by restricted intramolecular rotations: Aie and aiee effects. Chemical Communications, pages 70-72, 2007.

[7] Xi Cun Gao, Hong Cao, Ling Huang, Yan Yi Huang, Bao Wen Zhang, and Chun Hui Huang. Comparison of the electroluminescence and its related properties of two cyclopentadiene derivatives. Applied Surface Science, 210(3-4):183-189, 2003.

[8] Keiko Saito, Yoshimasa Bando, Atsushi Takahashi, and Hideaki Okamoto. Organic electroluminescence light-emitting device and production method thereof, 2016. US Patent US9385334, filed 5 February 2014 and issued 5 July 2016.

[9] Quansong Li and Llus Blancafort. A conical intersection model to explain aggregation induced emission in diphenyl dibenzofulvene. Chemical Communications, 49(53):5966, 2013.

[10] Rachel Crespo-Otero, Quansong Li, and Lluís Blancafort. Exploring potential energy surfaces for aggregation-induced emissionfrom solution to crystal. Chemistry An Asian Journal, 14(6):700-714, 2019. 
[11] Yingli Niu, Qian Peng, and Zhigang Shuai. Promoting-mode free formalism for excited state radiationless decay process with Duschinsky rotation effect. Science in China, Series B: Chemistry, 51(12):1153-1158, 2008.

[12] Xing-Liang Peng, Sergi Ruiz-Barragan, Ze-Sheng Li, Quan-Song Li, and Llus Blancafort. Restricted Access to a Conical Intersection to Explain Aggregation Induced Emission in Dimethyl Tetraphenylsilole. J. Mater. Chem. C, 4(14):2802-2810, 2016.

[13] Jeng Da Chai and Martin Head-Gordon. Long-range corrected hybrid density functionals with damped atom-atom dispersion corrections. Physical Chemistry Chemical Physics, 10(44):6615-6620, 2008.

[14] Riidiger Bauernschmitt and Reinhart Ahlrichs. Treatment of electronic excitations within the adiabatic approximation of time dependent density functional theory. Chemical Physics Letters, 256(4-5):454-464, 1996.

[15] Mark E. Casida, Christine Jamorski, Kim C. Casida, and Dennis R. Salahub. Molecular excitation energies to high-lying bound states from time-dependent density-functional response theory: Characterization and correction of the time-dependent local density approximation ionization threshold. Journal of Chemical Physics, 108(11):4439-4449, 1998.

[16] Eric R. Stratmann, Gustavo E. Scuseria, and Michael J. Frisch. An efficient implementation of time-dependent density-functional theory for the calculation of excitation energies of large molecules. The Journal of Chemical Physics, 109(19):8218-8224, 1998.

[17] Carole Van Caillie and Roger D. Amos. Geometric derivatives of excitation energies using SCF and DFT. Chemical Physics Letters, 308(3-4):249-255, 1999.

[18] Filipp Furche and Reinhart Ahlrichs. Adiabatic time-dependent density functional methods for excited state properties. Journal of Chemical Physics, 117(16):7433-7447, 2002.

[19] Ove Christiansen, Henrik Koch, and Poul Jørgensen. The second-order approximate coupled cluster singles and doubles model CC2. Chemical Physics Letters, 243(5-6):409-418, 1995.

[20] Christof Hättig and Andreas Köhn. Transition moments and excited-state first-order properties in the coupled-cluster model CC2 using the resolutionof-the-identity approximation. Journal of Chemical Physics, 117(15):69396951, 2002.

[21] Christof Hättig. Geometry optimizations with the coupled-cluster model CC2 using the resolution-of-the-identity approximation. Journal of Chemical Physics, 118(17):7751-7761, 2003. 
[22] Andreas Köhn and Christof Hättig. Analytic gradients for excited states in the coupled-cluster model CC2 employing the resolution-of-the-identity approximation. Journal of Chemical Physics, 119(10):5021-5036, 2003.

[23] Björn O. Roos, Andrzej J. Sadlej, Per Åke Malmqvist, Kerstin Andersson, and Krzysztof Wolinski. Second-order perturbation theory with a CASSCF reference function. The Journal of Physical Chemistry, 94(14):5483-5488, 1990 .

[24] Kerstin Andersson, Per Åke Malmqvist, and Björn O. Roos. Second-order perturbation theory with a complete active space self-consistent field reference function. The Journal of Chemical Physics, 96(2):1218-1226, 1992.

[25] Steven Vancoillie, Mickal G. Delcey, Roland Lindh, Victor Vysotskiy, Per-Åke Malmqvist, and Valera Veryazov. Parallelization of a multiconfigurational perturbation theory. Journal of Computational Chemistry, 34(22):1937-1948, 2013.

[26] Björn O. Roos, Peter R. Taylor, and Per E. M. Siegbahn. A complete active space SCF method (CASSCF) using a density matrix formulated super-CI approach. Chemical Physics, 48(2):157-173, 1980.

[27] Niclas Forsberg and Per Åke Malmqvist. Multiconfiguration perturbation theory with imaginary level shift. Chemical Physics Letters, 274(1-3):196204, 1997.

[28] Satoshi Maeda, Koichi Ohno, and Keiji Morokuma. Updated branching plane for finding conical intersections without coupling derivative vectors. Journal of Chemical Theory and Computation, 6(5):1538-1545, 2010.

[29] Francesco Aquilante, Jochen Autschbach, Rebecca K. Carlson, Liviu F. Chibotaru, Mickaël G. Delcey, Luca De Vico, Ignacio Fdez. Galván, Nicolas Ferré, Luis Manuel Frutos, Laura Gagliardi, Marco Garavelli, Angelo Giussani, Chad E. Hoyer, Giovanni Li Manni, Hans Lischka, Dongxia Ma, Per Åke Malmqvist, Thomas Müller, Artur Nenov, Massimo Olivucci, Thomas Bondo Pedersen, Daoling Peng, Felix Plasser, Ben Pritchard, Markus Reiher, Ivan Rivalta, Igor Schapiro, Javier Segarra-Martí, Michael Stenrup, Donald G. Truhlar, Liviu Ungur, Alessio Valentini, Steven Vancoillie, Valera Veryazov, Victor P. Vysotskiy, Oliver Weingart, Felipe Zapata, and Roland Lindh. Molcas 8: New capabilities for multiconfigurational quantum chemical calculations across the periodic table. Journal of Computational Chemistry, 37(5):506-541, 2016.

[30] TURBOMOLE V7.0 2015, a development of University of Karlsruhe and Forschungszentrum Karlsruhe GmbH, 1989-2007, TURBOMOLE GmbH, since 2007; available from http://www.turbomole.com.

[31] G. Evrard, P. Piret, G. Germain, and M. Van Meerssche. Structure cristalline et moléculaire du tétraphényl-1,2,3,4 cyclopentadiène. Acta Crystallographica Section B, 27(3):661-666, 1971. 
[32] Paolo Giannozzi, Stefano Baroni, Nicola Bonini, Matteo Calandra, Roberto Car, Carlo Cavazzoni, Davide Ceresoli, Guido L. Chiarotti, Matteo Cococcioni, Ismaila Dabo, Andrea Dal Corso, Stefano De Gironcoli, Stefano Fabris, Guido Fratesi, Ralph Gebauer, Uwe Gerstmann, Christos Gougoussis, Anton Kokalj, Michele Lazzeri, Layla Martin-Samos, Nicola Marzari, Francesco Mauri, Riccardo Mazzarello, Stefano Paolini, Alfredo Pasquarello, Lorenzo Paulatto, Carlo Sbraccia, Sandro Scandolo, Gabriele Sclauzero, Ari P. Seitsonen, Alexander Smogunov, Paolo Umari, and Renata M. Wentzcovitch. QUANTUM ESPRESSO: A modular and opensource software project for quantum simulations of materials. Journal of Physics Condensed Matter, 21(39):395-502, 2009.

[33] Stefan Dapprich, István Komaromi, K. Suzie Byun, Keiji Morokuma, and Michael J Frisch. A new ONIOM implementation in Gaussian98. Part I. The calculation of energies, gradients, vibrational frequencies and electric field derivatives. Journal of Molecular Structure Theochem, 461-462:121, 1999 .

[34] Lung Wa Chung, W. M.C. Sameera, Romain Ramozzi, Alister J. Page, Miho Hatanaka, Galina P. Petrova, Travis V. Harris, Xin Li, Zhuofeng Ke, Fengyi Liu, Hai Bei Li, Lina Ding, and Keiji Morokuma. The ONIOM Method and Its Applications. Chemical Reviews, 115(12):5678-5796, 2015.

[35] M. J. Frisch, G. W. Trucks, H. B. Schlegel, G. E. Scuseria, M. A. Robb, J. R. Cheeseman, G. Scalmani, V. Barone, G. A. Petersson, H. Nakatsuji, X. Li, M. Caricato, A. V. Marenich, J. Bloino, B. G. Janesko, R. Gomperts, B. Mennucci, H. P. Hratchian, J. V. Ortiz, A. F. Izmaylov, J. L. Sonnenberg, D. Williams-Young, F. Ding, F. Lipparini, F. Egidi, J. Goings, B. Peng, A. Petrone, T. Henderson, D. Ranasinghe, V. G. Zakrzewski, J. Gao, N. Rega, G. Zheng, W. Liang, M. Hada, M. Ehara, K. Toyota, R. Fukuda, J. Hasegawa, M. Ishida, T. Nakajima, Y. Honda, O. Kitao, H. Nakai, T. Vreven, K. Throssell, J. A. Montgomery, Jr., J. E. Peralta, F. Ogliaro, M. J. Bearpark, J. J. Heyd, E. N. Brothers, K. N. Kudin, V. N. Staroverov, T. A. Keith, R. Kobayashi, J. Normand, K. Raghavachari, A. P. Rendell, J. C. Burant, S. S. Iyengar, J. Tomasi, M. Cossi, J. M. Millam, M. Klene, C. Adamo, R. Cammi, J. W. Ochterski, R. L. Martin, K. Morokuma, O. Farkas, J. B. Foresman, and D. J. Fox. Gaussian16 Revision A.03, 2016. Gaussian Inc. Wallingford CT.

[36] Kenneth M. Merz, Wendy D. Cornell, Peter A. Kollman, David M. Ferguson, Piotr Cieplak, James W. Caldwell, Christopher I. Bayly, Ian R. Gould, David C. Spellmeyer, and Thomas Fox. A Second Generation Force Field for the Simulation of Proteins, Nucleic Acids, and Organic Molecules. Journal of the American Chemical Society, 117(19):5179-5197, 2005.

[37] Juan Aragó and Alessandro Troisi. Dynamics of the excitonic coupling in organic crystals. Physical Review Letters, 114(2):1-5, 2015. 
[38] M. Dommett, M. Rivera, and R. Crespo-Otero. How Inter- and Intramolecular Processes Dictate Aggregation-Induced Emission in Crystals Undergoing Excited-State Proton Transfer. J. Phys. Chem. Lett., 8(24):6148-6153, 2017.

[39] J. R. Reimers. A practical method for the use of curvilinear coordinates in calculations of normal-mode-projected displacements and duschinsky rotation matrices for large molecules. Journal of Chemical Physics, 115(20):9103-9109, 2001.

[40] Mario Barbatti, Giovanni Granucci, Maurizio Persico, Matthias Ruckenbauer, Mario Vazdar, Mirjana Eckert-Maksić, and Hans Lischka. The onthe-fly surface-hopping program system Newton-X: Application to ab initio simulation of the nonadiabatic photodynamics of benchmark systems. Journal of Photochemistry and Photobiology A: Chemistry, 190(2-3):228$240,2006$.

[41] Mario Barbatti, Matthias Ruckenbauer, Felix Plasser, Jiri Pittner, Giovanni Granucci, Maurizio Persico, and Hans Lischka. Newton-X: a surfacehopping program for nonadiabatic molecular dynamics. Wiley Interdisciplinary Reviews: Computational Molecular Science, 4(1):2014, 2013.

[42] Rachel Crespo-Otero and Mario Barbatti. Recent advances and perspectives on nonadiabatic mixed quantumclassical dynamics. Chemical Reviews, 118(15):7026-7068, 2018.

[43] Mario Barbatti, Adélia J. A. Aquino, and Hans Lischka. The UV absorption of nucleobases: semi-classical ab initio spectra simulations. Physical Chemistry Chemical Physics, 12(19):49594967, 2010.

[44] Rachel Crespo-Otero and Mario Barbatti. Spectrum simulation and decomposition with nuclear ensemble: Formal derivation and application to benzene, furan and 2-phenylfuran. Theoretical Chemistry Accounts, 131(6):1237, 2012.

[45] Yingli Niu, Qian Peng, Chunmei Deng, Xing Gao, and Zhigang Shuai. Theory of excited state decays and optical spectra: Application to polyatomic molecules. Journal of Physical Chemistry A, 114(30):7817-7831, 2010.

[46] Han Nie, Kun Hu, Yuanjing Cai, Qian Peng, Zujin Zhao, Rongrong Hu, Junwu Chen, Shi-Jian Su, Anjun Qin, and Ben Zhong Tang. Tetraphenylfuran: aggregation-induced emission or aggregation-caused quenching? Mater. Chem. Front., 1(6):1125-1129, 2017.

[47] Shiwei Yin, Qian Peng, Z. Shuai, Weihai Fang, Yan Hua Wang, and Yi Luo. Aggregation-enhanced luminescence and vibronic coupling of silole molecules from first principles. Physical Review B - Condensed Matter and Materials Physics, 2006. 
[48] M Kasha, H R Rawls, and M Ashraf El-Bayoumi. The Exciton Model in Molecular Spectroscopy. Pure and Applied Chemistry, 11(3-4):371-392, 1965 .

[49] Wenqiang Li, Lili Zhu, Qiang Shi, Jiajun Ren, Qian Peng, and Zhigang Shuai. Excitonic coupling effect on the nonradiative decay rate in molecular aggregates: Formalism and application. Chemical Physics Letters, 683:507 $-514,2017$.

[50] Jiri Sturala, Marc K. Etherington, Aisha N. Bismillah, Heather F. Higginbotham, William Trewby, Juan A. Aguilar, Elizabeth H. C. Bromley, Alyssa-Jennifer Avestro, Andrew P. Monkman, and Paul R. McGonigal. Excited-state aromatic interactions in the aggregation-induced emission of molecular rotors. Journal of the American Chemical Society, 139(49):17882-17889, 2017.

[51] Michael Dommett and Rachel Crespo-Otero. Excited state proton transfer in 2'-hydroxychalcone derivatives. Phys. Chem. Chem. Phys., 19:24092416, 2017.

[52] Xing Gao, Qian Peng, Yingli Niu, Dong Wang, and Zhigang Shuai. Theoretical insight into the aggregation induced emission phenomena of diphenyldibenzofulvene: a nonadiabatic molecular dynamics study. Phys. Chem. Chem. Phys., 14:14207-14216, 2012.

[53] Felix Plasser, Mario Barbatti, Adélia J.A. Aquino, and Hans Lischka. Excited-state diproton transfer in [2,2'-bipyridyl]-3,3'-diol: The mechanism is sequential, not concerted. Journal of Physical Chemistry A, 113(30):8490-8499, 2009.

[54] L. Kurtz, A. Hofmann, and R. De Vivie-Riedle. Ground state normal mode analysis: Linking excited state dynamics and experimental observables. Journal of Chemical Physics, 114(14):6151-6159, 2001.

[55] Mario Barbatti, Zhenggang Lan, Rachel Crespo-Otero, Jaroslaw J. Szymczak, Hans Lischka, and Walter Thiel. Critical appraisal of excited state nonadiabatic dynamics simulations of $9 \mathrm{H}$-adenine. The Journal of Chemical Physics, 137(22):22A503, 2012.

[56] Felix Plasser, Rachel Crespo-Otero, Marek Pederzoli, Jiri Pittner, Hans Lischka, and Mario Barbatti. Surface hopping dynamics with correlated single-reference methods: 9H-adenine as a case study. Journal of Chemical Theory and Computation, 10(4):1395-1405, 2014. 